\title{
Quinoa - The Health Food - A Boon for Vegans and Vegetarians
}

\section{Aditi Jain ${ }^{1}$, Faith B Varma ${ }^{2}$ and Ajit Varma ${ }^{3 *}$}

${ }^{1}$ Amity Institute of Microbial Technology, Amity University, Noida, UP, India

${ }^{2}$ St. Agnes Academy, Houston Texas, USA

${ }^{3}$ Former Pro Vice Chancellor, Science and Technology International, Distinguished Scientist and Professor of Eminence, Amity Institute of Microbial Technology and Vice Chairman, Amity Science, Technology and Innovation Foundation (ASTIF), Amity University, Uttar Pradesh, India

*Corresponding Author: Ajit Varma, Former Pro Vice Chancellor, Science and Technology International, Distinguished Scientist and Professor of Eminence, Amity Institute of Microbial Technology and Vice Chairman, Amity Science, Technology and Innovation Foundation (ASTIF), Amity University, Uttar Pradesh, India.

Received: July 11, 2019; Published: July 19, 2019

DOI: $10.31080 /$ ASPS.2019.03.0346

Quinoa and its Origin

Quinoa (pronounced as 'Keen-wa') is an annual seed producing grain crop. It is a pseudo-cereal that is botanically related to Spinach - Chenopodium quinoa (Figure 1). It is a wheat-free alternative to starchy grains which is rich in several important nutrients that come forward as a super-food for vegans and vegetarians that lack various nutrients such as essential amino acids, minerals, etc., in their normal, animal product - free diet. It takes less time to cook and comes in different varieties having different taste.

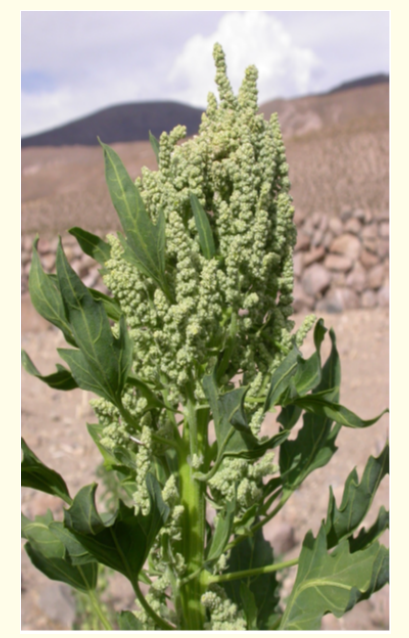

Figure 1: A typical Quinoa Plant at flowering stage (C.F. - Bazile Didier) [1].

Quinoa is originally a native crop of the Andean region of northwest South America, but due to an increase in World trade, its cultivation has spread to more than 70 countries around the world.
It has been grown in South America (Peru, Bolivia and Chile) for thousands of years. Earlier, about 5.2-7 years it was used to feed livestock, later 3-4 thousand years ago it entered the human diet, mainly in Peru and Bolivia. Now, owing to its nutritional content, health benefits, adaptability to different climates and low-cost of cultivation, it is emerging as a preferred food item over wheat and rice.

\section{Nutritional content}

Quinoa is naturally gluten-free. One cup of cooked quinoa, weighing 185 grams (g), contains:

- 222 calories

- $8.14 \mathrm{~g}$ of protein

- $5.2 \mathrm{~g}$ of fiber

- $\quad 3.55 \mathrm{~g}$ of fat, of which $0.42 \mathrm{~g}$ is saturated

- $\quad 39.4 \mathrm{~g}$ of carbohydrate

- Magnesium - 30 percent recommended daily allowance (RDA)

- Manganese - 30 percent RDA

- Folate - 19 percent RDA

- Phosphorous - 28 percent RDA

- Copper - 18 percent RDA

- Iron - 15 percent RDA

- Zinc - 13 percent RDA

- Potassium - 9 percent RDA

One cup also contains more than 10 percent of the RDA of the vitamins B-1, B-2, and B-6, and traces of vitamin E, B3, and calcium.

Additionally, quinoa contains a healthy dose of fatty acids: "About 25 percent of quinoa's fatty acids come in the form of oleic acid, a heart-healthy monounsaturated fat, and about 8 percent 
comes in the form of alpha-linolenic acid (ALA), the omega-3 fatty acid most commonly found in plants".

Melinda Lund, M.S., R.D., a registered dietitian and medical nutrition therapist

\section{Zinc and human health}

Zinc is known to play a critical role in normal functioning of body and is integrated with several enzyme systems. Adequate dietary intake of zinc has been shown to exert ameliorating effect on the skin, and this attenuates the likelihood of restricted linear growth in young children. Neuro-behavioral disturbances among infants and repeated infections are common among zinc-deficient people of all ages. Pregnant women with zinc deficiency are at the risk of complicated pregnancy outcomes [2]. This is further complicated by the fact that Zinc deficiency is one of the major factors limiting the yield of agricultural crops. This necessitated the need to fortify Black Rice with Zinc using Zinc oxide nanoparticles. It is believed that Black Rice augmented with Zinc would not only ameliorate the health of the marginalized sections of the Indian populace but also pave the way to improve the agricultural yield thereby sustainably improving the carrying capacity of the arable land [3].

Content of essential amino acids (EAA) in Quinoa in comparison to the values recommended by FAO (in grams per 100 grams of protein)

\begin{tabular}{|l|c|c|}
\hline & FAO Recommendation & Quinoa \\
\hline Isoleucine & 3.0 & 4.9 \\
\hline Leucine & 6.1 & 6.6 \\
\hline Lysine & 4.8 & 6.0 \\
\hline Methionine & 2.3 & 5.3 \\
\hline Phenylalanine & 4.1 & 6.9 \\
\hline Threonine & 2.5 & 3.7 \\
\hline Tryptophan & 0.66 & 0.9 \\
\hline Valine & 4.0 & 4.5 \\
\hline
\end{tabular}

Table 1: Amino acid content of Quinoa (C.F. - M.J. Koziol) [4].

\section{Types of quinoa}

There are over 120 different varieties of Quinoa as defined by the Whole Grains Council but the most common Quinoa colours found across grocers and markets are white (also considered ivory or yellow quinoa), red and black (Figure 2).

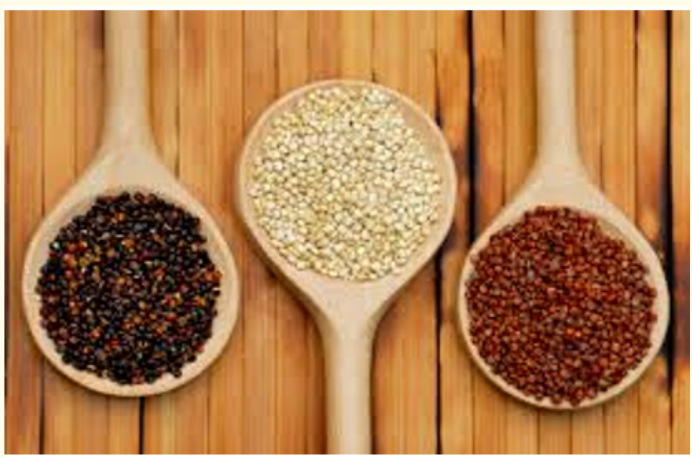

Figure 2: Varieties of Quinoa white, red and black (C.F. - Alia Hoyt).

Nutritional values among the three types are almost similar, with minor differences such as black quinoa having the lowest fat and iron value.

\begin{tabular}{|l|l|l|l|}
\hline & White Quinoa & Red Quinoa & Black Quinoa \\
\hline Calories & 355 & 380 & 370 \\
\hline Fat & $6 \mathrm{~g}$ & $7.2 \mathrm{~g}$ & $3.3 \mathrm{~g}$ \\
\hline Protein & $13 \mathrm{~g}$ & $14 \mathrm{~g}$ & $11 \mathrm{~g}$ \\
\hline Iron & $90 \%$ & $50 \%$ & $20 \%$ \\
\hline
\end{tabular}

Table 2

Quinoa also has high content of two types of flavonoids (antioxidants) quercetin and kaempferol. Allowing the seeds to sprout seems to increase the antioxidant content even further.

Where taste is concerned, red and black varieties tend to have an earthier flavor with black having an additional sweeter taste as compared to the white variety. When cooked, white quinoa appears fluffier, whereas both red and black quinoa tend to be a bit more crunchy in texture.

\section{Where is quinoa grown in India?}

In India, as farmers and people are becoming more aware and receiving food and agricultural education, crops having improved quality and nutritional value have increased both in productivity and consumer demand. Mainly, the States of Andhra Pradesh, Uttarakhand and Rajasthan are emerging as the main cultivators of Quinoa in India. 
Various conditions but some key breeding objectives in Quinoa cultivation

- $\quad$ Seed yield

- Heat tolerance

- Resistance to downy mildew

- Low saponin content

- Drought tolerance

- $\quad$ Early maturity

- $\quad$ Pre-harvest sprouting resistance

- Nutritional value and flavour

\section{Cooking protocol of Quinoa}

Ingredients

- 1 cup quinoa, $(194 \mathrm{~g})$

- 2 cups water, $(480 \mathrm{ml})$

- $1 / 2$ teaspoon kosher salt, (3g)

- Vegetables (optional). Most common vegetables added are carrot, peas, beans and broccoli. Addition of Cashew nuts or peanuts adds flavour.

\section{Instructions}

- $\quad$ Place quinoa in a medium-sized bowl and cover with cold water. Soak for 2 minutes.

- Transfer soaked quinoa to a fine-mesh sieve. Rinse quinoa under running cold water for 30 seconds until water runs clear

- Shake the sieve to remove excess water.

- Transfer rinsed quinoa to a medium-sized pot fitted with a lid. Add vegetables according to the taste.

- $\quad$ Add 2 cups of cold water and salt to the pot.

- Bring water to a boil over high heat.

- Once the water boils, immediately reduce heat to mediumlow.

- Cover pot and simmer until quinoa absorbs all of the water and is tender about 15 to 20 minutes. If the quinoa still looks wet, continue to cook, checking every 5 minutes.

- Turn off heat and let stand, covered 5 to 10 minutes. Fluff with a fork to separate the grains.

Note

Toasting Option: Once quinoa is rinsed, it can be sautéed in 2 tablespoons olive oil or any other vegetable oil over medium heat for a few minutes before adding in water add salt. This will accentuate the slightly bitter and nutty flavors of the quinoa.
Average production per acre of land

According to Mr. Manoj Upadhyaya (Pune), in India it is available for Rs. $1800-2000 / \mathrm{kg}$. $2 \mathrm{~kg}$ seeds are required for 1 acres quinoa farming. Quinoa is a self-resistant crop and less prone to insect or microorganism attack. "At the moment it is free from pest but may be Indian insect will start stacking the crop soon, then 'gomutra' and 'neem leaves extract' can be used as preventive measures".

Yield per acre is about $450 \mathrm{~kg}$ but may range from $600-700 \mathrm{~kg} /$ acre.

Strategies to enhance the yield and value addition to the seeds is promised by interaction with Symbiotic fungus Piriformospora indica (Serendipita indica) and ZnO nanoparticles singly and/or in combination.

$P$. indica is a plant beneficial fungus that supports plant growth during its mutualistic symbiotic relationship by root colonization which results in early flowering, higher seed yield, alteration in the secondary metabolites, and adaptation to abiotic and biotic stresses. $P$. indica - infested roots also show antioxidant capacity. The fungus also induces systemic disease resistance in plants.

Mass multiplication of $P$. indica on cheap, reproducibly and reliable Jaggery (Gur) as sole Carbon energy source and P. indica fungus showing fluorescent pear shaped spores (Figure 3).

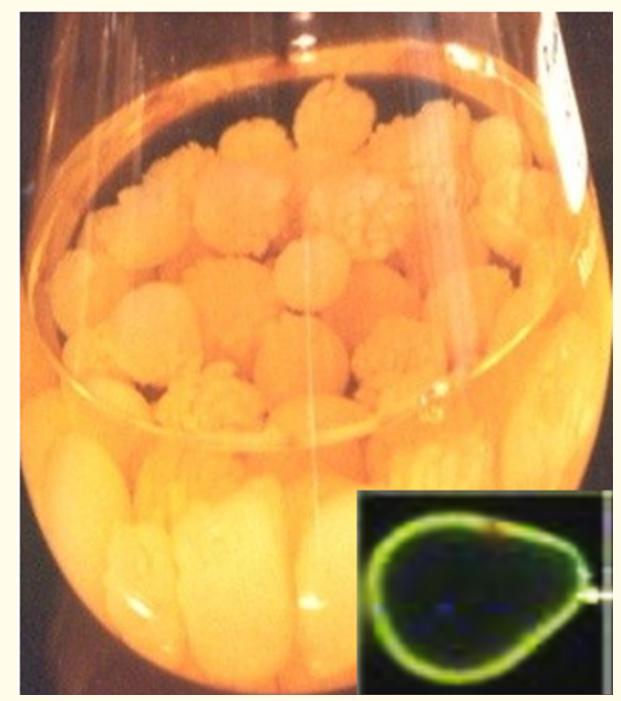

Figure 3: Cultivation of Piriformospora indica on eco-friendly medium containing $4 \%$ jaggery (obtained from Saccharum officinarum) (944/DEL/2012 patent). 
Treatment with ZnO Nanoparticles.

\section{Synthesis of nano zinc materials}

$\mathrm{ZnO}$ nanorods were prepared by a mechanical-assisted thermal decomposition process. Zinc acetate dihydrate [ $\left.\mathrm{Zn}\left(\mathrm{CH}_{3} \mathrm{COO}\right) 22 \mathrm{H}_{2} \mathrm{O}\right]$ was used as precursor for synthesis of zinc oxide nanorods. In the synthesis process, zinc acetate dihydrate (5 g) was ground in a mortar and pestle for $45 \mathrm{~min}$ then loaded into an alumina crucible, which was then heated in a programmable muffle furnace (at ramp rate of $4 \mathrm{C} / \mathrm{min}$ ) at $275^{\circ} \mathrm{C}$ for $4 \mathrm{~h}$ to obtain $\mathrm{ZnO}$ nanopowders. The obtained powders were then washed twice with distilled water, followed by drying in an oven at $80^{\circ} \mathrm{C}$ for 8 hours.

The nanoparticles were spherical, oblong and rod in shape (Figure 4). The diameter varied from 9.6-25.5 $\mathrm{nm}$ in size [5].

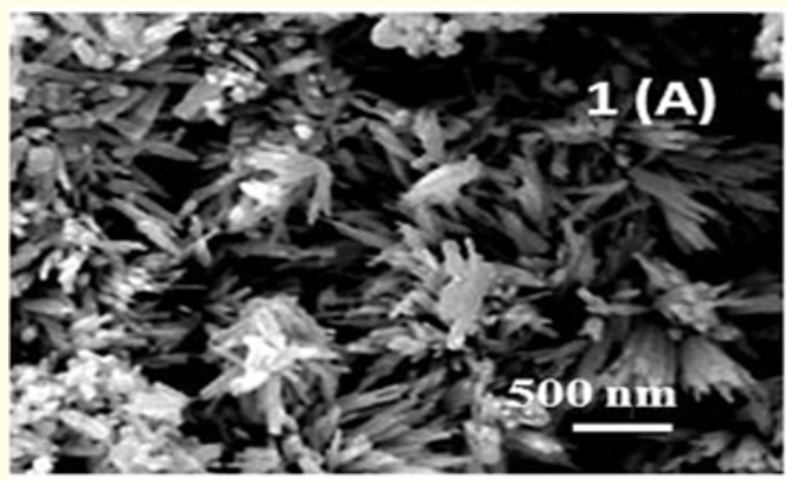

Figure 4: Scanning electron microscopy (SEM) micrograph of pure $\mathrm{ZnO}$ nanorods.

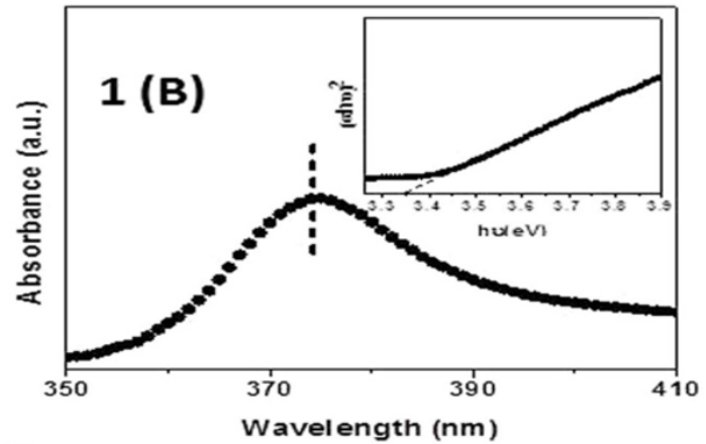

Figure 5: Tauc's plot and UV-Vis absorption spectra of pure $\mathrm{ZnO}$ nanorods.
Figure 6 Indicates the morphology of the spores with and without treatment with $\mathrm{ZnO}$ nanoparticles as observed under confocal microscope (Model Nikon A1).

For confocal based analysis of $P$. indica spores the technique adopted by Hilbert et al. 2013 with slight modifications was used. $0.002 \%$ freshly prepared Tween twenty solution was autoclaved and stored at $4^{\circ} \mathrm{C} .1 \mathrm{ml}$ of the solution was added to $P$. indica culture plate and spores were scraped out with the help of spreader and collected in $2 \mathrm{ml}$ micro-centrifuge tubes followed by centrifugation at $5000 \mathrm{G}$ for 15 minutes. The plate was collected and mixed with $1 \mathrm{ml}$ autoclaved distilled water while the supernatant was discarded. The spores were stained with WGA Alexa 488 conjugated dye. Approximately, $5 \mu \mathrm{l}$ of the dye $(10 \mu \mathrm{g}$ per ml conc) was used for staining $500 \mu \mathrm{l}$ of spores and was subjected to confocal microscopy. The images were captures using confocal microscope (Model Nikon A1) under magnification of 40X. The confocal setting WGA - Alexa 488 conjugated dye was as follows:

- $\quad$ Excitation - 488nm, emission 515

- The shelf life of $\mathrm{ZnO}$ embedded fungal spores is prolonged due to presence of nanomaterials.

Comparative analysis

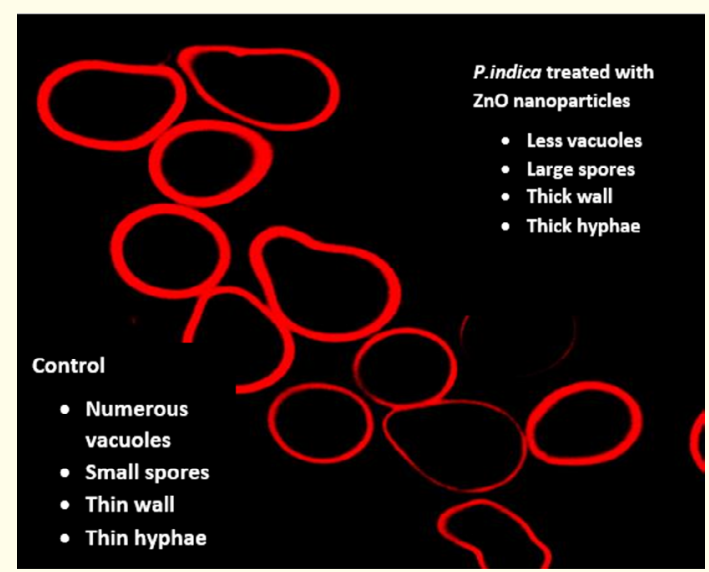

Figure 6: Morphology and essential characteristics of P.indica spores with and without $\mathrm{ZnO}$ particle treatment.

\section{Ongoing research}

The experiments on interaction of Zinc oxide ( $\mathrm{ZnO}$ ) nanoparticles and the beneficial fungus) with quinoa seeds are in progress under temperature and environment control greenhouse condi- 
tions. With a sole objective to enhance the yield and value addition to the seeds (flavonoids quercetin and kaempferol). The aim is also focused on keeping the crop disease free [6-20].

\section{Acknowledgements}

Authors are thankful to NASF (ICAR) and DST Nano-mission for partial financial support.

\section{Bibliography}

1. Bazile Didier. Creating a shared vision and action plan for the future of quinoa beyond its origins (2016).

2. Jillian Levy CHHC. "10 Powerful Zinc Benefits, Including Fighting Cancer”. Dr. Axe (2018).

3. Varma., et al. "Zinc oxide nanorods for fungus productivity and broccoli (Brassica oleracea var. botrytis) development (2019).

4. Koziol MJ. Chemical composition and nutritional evaluation of quinoa (Chenopodium quinoa wild.) (1992).

5. Singhal Uma., et al. "Impact of Synergistic Association of ZnONanorods and Symbiotic Fungus Piriformospora indica DSM 11827 on Brassica oleracea var. botrytis (Broccoli)". Frontiers in Microbiology 17 (2017): 1-6.

6. Collyns Dan. "IQuinoa brings riches to the Andes". The Guardian. London (2013).

7. Gavin Jessica. How to cook quinoa (2018).

8. Levin Jo. The health benefits of quinoa (2018).

9. Masterbroek HD., et al. "Occurrence of sapogenins in leaves and seeds of Quinoa (Chenopodium quinoa Willd)". Journal of the Science of Food and Agriculture 80 (2000): 152-156.

10. Mehra Purva. Grain shower: Indian farmers are now growing quinoa (2016).

11. Piriformospora.

12. Quinoa.

13. Steinfeld Katie. White vs. Red vs. Black Quinoa - What's the Difference? (2014).
14. Tamanna., B., et al. "Biosynthesis of zinc oxide nanoparticles from Azadirachta indica for antibacterial and photocatalytic applications". Material Science in Semiconductor Processing 32 (2015): 55-61.

15. Tamanna., et al. "A comparative study of pure and copper (Cu)doped ZnO nanorods for antibacterial and photocatalytic applications with their mechanism of action". Journal of Nanoparticle Research 17 (2015): 1-11.

16. Uma Attri., et al. "Zinc oxide nanorods for fungus productivity and broccoli (Brassica oleracea var.botrytis) development". International Classification (2018).

17. Upadhyaya Manoj. Quinoa, a new profitable crop (2015).

18. Varma., et al. "Piriformospora indica: A Novel Plant GrowthPromoting Mycorrhizal Fungus”. Springer (2012).

19. Varma., et al. "A nanomaterial based culture medium for microbial growth enhancement (2015).

20. Ware Megan. Health benefits of quinoa (2018).

\section{Volume 3 Issue 8 August 2019 \\ (C) All rights are reserved by Ajit Varma., et al.}

